\title{
Self-Motion and the Hippocampal Spatial Metric
}

\author{
Alejandro Terrazas, ${ }^{1}$ Michael Krause, ${ }^{1}$ Peter Lipa, ${ }^{1}$ Katalin M. Gothard, ${ }^{2}$ Carol A. Barnes,,${ }^{1,3,4}$ and \\ Bruce L. McNaughton ${ }^{1,2,3}$ \\ ${ }^{1}$ Arizona Research Laboratories Division of Neural Systems, Memory and Aging, Departments of ${ }^{2}$ Physiology, ${ }^{3}$ Psychology, and ${ }^{4}$ Neurology, University of \\ Arizona, Tucson, Arizona 85724
}

Self-motion signals are sufficient for animal navigation ("path integration") and for updating hippocampal location-specific firing. The contributions of ambulatory, vestibular, and optic self-motion signals to CA1 unit activity and EEG were studied while rats either walked or drove a car between locations on a circular track (referred to as WALK and CAR, respectively) or experienced pseudomotion, in which the animal was stationary and the environment was rotated (WORLD). Fewer pyramidal cells expressed place fields during CAR and those that did exhibited substantially larger place fields. The number of theta cycles required to traverse a place field increased, whereas the slope of the theta phase of firing versus position function was reduced. The presence and/or location of place fields were not well correlated between conditions. These effects were even more accentuated during WORLD. These results are not explainable by a simple "smearing out" of place fields but, in terms of size of place fields relative to the track size, are comparable with what would be observed if the track circumference was reduced and the animal moved around it at a correspondingly slower speed. Theta (and its $14-18 \mathrm{~Hz}$ harmonic) power were dependent on velocity, but the gain of this function was substantially reduced during CAR and WORLD, again as if the rat were moving more slowly. The spatial scale over which the hippocampal population vector is updated appears to be derived primarily from the gain of a self-motion velocity signal with approximately equal components derived from ambulation, vestibular, and optic-flow signals.

Key words: spatial navigation; path integration; vestibular; place cells; theta rhythm; ensemble recordings

\section{Introduction}

Hippocampal neural activity can be highly correlated with the animal's position in the environment. Although external sensory cues play an important role in establishing the spatial and directional reference for this system, the hippocampal positional correlate can be updated through the process of path integration (Mittelstaedt and Mittelstaedt, 1980) of ambulatory, optic, and vestibular self-motion signals (Quirk et al., 1990; Markus et al., 1994; Gothard et al., 1996; McNaughton et al., 1996); however, the specific contributions of these different self-motion signals to the hippocampal spatial metric remain unknown.

In their original treatise, O'Keefe and Nadel (1979) postulated that the hippocampal theta rhythm (a prominent 7-9 Hz oscillation in the hippocampal electroencephalogram (EEG) that accompanies the animal's displacements in space) provides a distance "metric" (standard of measurement) to the hippocampal cognitive map. Several experiments have provided some support for this hypothesis. For example, the spectral power of the theta rhythm (Czurko et al., 1999) and population firing rate (McNaughton et al., 1983a; Czurko et al., 1999; Ekstrom et al., 2001) are dependent on the running speed of the rat. Moreover, when

Received Feb. 20, 2005; revised July 22, 2005; accepted July 25, 2005.

This work was supported by National Institutes of Health Grants AG012609 and NSO20331. We thank Kate Poneta, Kim Bohne, and Jennifer Dees for assistance with data collection and analysis and Michelle Carroll for assistance with manuscript preparation.

Correspondence should be addressed to Dr. Bruce L. McNaughton, University of Arizona, Neural Systems, Life Sciences North Building, Room 384, Tucson, AZ 85724. E-mail: bruce@nsma.arizona.edu.

DOI:10.1523/JNEUROSCI.0693-05.2005

Copyright $\odot 2005$ Society for Neuroscience $\quad$ 0270-6474/05/258085-12\$15.00/0 limb movements are rendered impossible by restraining the animal in a towel, the theta rhythm is greatly attenuated and place cells become silent (Foster et al., 1989).

To dissociate the specific influences of ambulatory, vestibular, and optic-flow self-motion signals on the hippocampal spatial metric, hippocampal EEG and CA1 pyramidal cells ("place cells") were recorded while rats either walked (WALK) or drove themselves between locations on a circular track, by pressing and holding down a GO button on a small car (CAR). The latter condition eliminated ambulatory signals, leaving optic and vestibular signals intact. In follow-up experiments, a condition was added in which the car remained stationary while the entire cylindrical environment was rotated around the rat (WORLD). This latter condition eliminated both ambulatory and vestibular selfmotion signals.

Parts of this paper have been published previously in abstract form (Terrazas et al., 2001).

\section{Materials and Methods}

Recording method

Multiple single units were recorded from the CA1 region of the dorsal hippocampus using "tetrodes" (McNaughton et al., 1983a; Recce and O'Keefe, 1989), four-channel electrodes constructed by twisting together four strands of insulated $13 \mu \mathrm{m}$ nichrome wire (H. P. Reid, Neptune, NJ). Each animal was implanted with an array of 14 independently adjustable microdrives (HyperDrive) containing 12 tetrodes and two additional electrodes, which served as reference (in or near corpus callosum) and a theta probe (located at or near the hippocampal fissure). Each microdrive consisted of a drive screw coupled by a nut to a guide cannula. A full turn of the screw advanced the tetrode $318 \mu \mathrm{m}$. The hyperdrive, 
implantation methods, and the parallel recording technique have been described in detail previously (Wilson and McNaughton, 1993; Gothard et al., 1996).

\section{Surgery}

Six male Fischer 344 rats (age range 10-18 months; Charles River Laboratories, Wilmington, MA) were used as experimental subjects. Animal care, surgical procedures, and killing were performed in accordance with the National Institutes of Health and University Animal Care guidelines for the use of vertebrate animals in research. The animals were housed individually in Plexiglas containers and maintained on a reversed $12 \mathrm{~h}$ light/dark cycle in a colony room. Subjects had access to water ad libitum and were weighed, handled, and checked for overall health daily. After surgical recovery $(\sim 5 \mathrm{~d})$, animals were maintained at $75-80 \%$ of their ad libitum weights until the conclusion of the experiments.

During electrode implantation, rats were anesthetized using sodium pentobarbital anesthesia (Nembutal, $40 \mathrm{mg} / \mathrm{kg}$, i.p.; Abbott Laboratories, Abbott Park, IL) and placed in a stereotaxic frame (David Kopf Instruments, Tujunga, CA). Small doses of supplemental methoxyflurane (Metofane; Pitman-Moore Pharmaceuticals, Chicago, IL) were administered by inhalation as required to maintain a state of deep anesthesia throughout the surgery.

Just before insertion of the HyperDrive, bilateral stimulation electrodes were implanted with their tips aimed at the medial forebrain bundle (MFB). In two of the animals, two additional electrodes were implanted contralaterally, aiming for the hippocampal fissure and alveus/ stratum oriens, respectively. This was done to facilitate optimal differential recording of the theta component of the EEG. The leads of these additional electrodes were later connected to the HyperDrive circuit board and then attached to the dental acrylic mass surrounding the HyperDrive post.

Immediately after surgery, 30,000 U of penicillin (Bicillin; WyethAyerst, Philadelphia, PA) was administered intramuscularly to each hindlimb, and the animals were allowed to recover for $4-8 \mathrm{~h}$ in an incubator. After recovery from anesthesia, $25 \mathrm{mg}$ of acetaminophen (Children's Tylenol Elixir; McNeil, Fort Washington, PA) was administered orally for analgesia. The rat was then placed in a clean cage with a surgical sheet. An additional $2.7 \mathrm{mg} / \mathrm{ml}$ acetaminophen dissolved in the drinking water was provided for $3 \mathrm{~d}$ after surgery.

\section{Behavioral apparatuses}

Two apparatuses were used in the course of these experiments. The rat train apparatus (RTA) consisted of a modified toy-train car (Lionel Trains, Chesterfield, MI) riding on top of a $130-\mathrm{cm}$-diameter G-scale circular track. The track was bolted to a round $150 \mathrm{~cm}$ diameter piece of plywood and placed on top of a cinderblock platform at a height of $60 \mathrm{~cm}$. The car was constructed by bolting a metal platform to the chassis of the toy-train engine. The entire apparatus was placed in the center of the recording room in plain view of prominent and stable distal cues, including the door and objects mounted on the walls. A video camera was mounted in the center of the room for tracking position.

The rat controlled vehicle (RCV) consisted of two independently movable, concentric axes (WORLD and CAR) driven by stepper motors (Oriental Motors, City of Industry, CA) controlled by either a rat-activated lever on the vehicle or the experimenter, such that the vehicle could either move around the track, or the entire "world," including floor, track, and curtains, could be rotated while the rat was on the stationary vehicle, thus providing the same optic-flow profile that would have occurred if the vehicle had actually been in motion. The stepper motors were attached to a modified circular metal patio table with mounted bearings (Browning Bearings and Chain, Monroe, NC). The entire maze was attached to the WORLD axis, including the large circular wooden platform $(140 \mathrm{~cm}$ diameter) with attached black curtains, the fabric ceiling, and the running track (also $130 \mathrm{~cm}$ diameter, as in the RTA above). The "vehicle" consisted of a small platform mounted over the track and connected to the CAR axis via a rigid radial spoke. Caster wheels mounted to the patio table provided stabilization of the maze. The positions of both axes were recorded as pulses by optical encoders (U.S. Digital, Vancouver, WA). The axis motors, task administration, and time stamping were controlled by an 80486 personal computer running the Windows 98 operating system (Microsoft, Redmond, WA). A single white cloth $(1 \times 0.5 \mathrm{~m})$ and directional light source were mounted to the inside surface of the circular curtains with a separation of $145^{\circ}$. A small opening in the top of the ceiling allowed for the installation of a video camera to image the inside of the apparatus for the purpose of positional tracking.

\section{Behavioral tasks}

The velocity of the vehicle was set to reach a top speed of $20 \mathrm{~cm} / \mathrm{s}$ by placing a small load on the car and engaging the motor for timed laps. The vehicle used in the RTA had no mechanism to control the acceleration or deceleration of the vehicle. The RCV used a 3 s linear acceleration/ deceleration control provided by the stepper motors. Neither apparatus enabled the rat to control the speed of the car.

Behavior was reinforced either by food or MFB stimulation. The subject was trained to push a lever, thereby engaging the car in forward motion. This behavior was shaped so that the subject made longer trajectories until the distance traveled was consistently $>30 \mathrm{~cm}$. The subject was not allowed to get off of the car. At this time, reinforcement became contingent on the subject reaching broad regions surrounding the goals.

Subjects were trained to perform one of two driving tasks that required stopping at various unmarked locations on the circular track. On the RTA, subjects drove from one of three starting locations to a single goal area subtending $30^{\circ}$ of arc on the track. After arrival at the goal, the subjects were rewarded and then driven passively to the next pseudorandomly chosen starting location. This task was abandoned in the RCV experiments in favor of a task that could be administered using the overhead video monitor and automated procedures. In the RCV experiments, subjects drove between two stopping locations. In both cases, care was taken to avoid "beacons" and other landmark cues at the goal locations. Subjects on both apparatuses were also sometimes driven around the track several times under the control of the investigator. This condition was termed EXPRESS.

For the RTA experiments, movement conditions were administered in blocks. The experiments on the RTA were primarily concerned with the characterization of the spatial representation when ambulatory selfmotion cues were eliminated; therefore, the majority of the trials were CAR trials. The subject was placed on the track for the WALK condition only after completing 60-100 laps of the CAR condition. This typically resulted in 10-20 laps of WALK as a control. In one of the experiments conducted on the RTA, the subject performed two blocks of CAR trials with a block of WALK trials in between.

The RCV experiments were primarily concerned with the comparison of CAR and WORLD movement conditions and whether place fields and EEG were further degraded when vestibular self-motion signals were eliminated. Subjects were shaped first to drive in the CAR condition and were recorded from for several days. The subjects were then transitioned to the WORLD condition for several days. For two of the WORLD datasets included in the data used in these analyses, the subject also walked several laps at the end of the recording session. These data were used for some within-session EEG analyses (see Fig. 2) but did not provide adequate sampling for within-session single-unit comparisons. Only recordings with 10-20 laps were included in these analyses.

\section{Data acquisition}

Because of the continual advancement of the neural data acquisition technology, two different configurations of the data acquisition system were used for the RTA and RCV experiments. The earlier RTA experiments used eight 80486 personal computers running Discovery data acquisition software (BrainWave, Broomfield, CO). Hippocampal EEG was bandpass filtered between 1 and $100 \mathrm{~Hz}$ and recorded at $250 \mathrm{~Hz}$. The position of infrared diodes on the head stage was sampled at $20 \mathrm{~Hz}$ using a video tracking system (Dragon Tracker' Dragon Systems, Boulder, CO). The more recent RCV experiments used a 48-channel Cheetah system (Neuralynx, Tucson, AZ). Single units were bandpass filtered between $600 \mathrm{~Hz}$ and $6 \mathrm{kHz}$ and recorded at $32 \mathrm{kHz}$. EEG was bandpass filtered at $1-300 \mathrm{~Hz}$ and recorded at $2.4 \mathrm{kHz}$. 


\section{Data analysis}

EEG recordings. Trajectories were segregated according to the various experimental conditions. The power spectrum was computed for the EEG of each epoch using the Matlab SPECGRAM function (MathWorks, Natick, MA). Relative theta power, defined as the ratio of power in the theta band $(7-9 \mathrm{~Hz})$ divided by the power in the delta band $(1-4 \mathrm{~Hz})$, was computed for the power spectrum of each trajectory $>4 \mathrm{~s}$ in duration. The ratio was used to adjust for variation in total EEG power attributable to electrode location in different sessions and across animals (Csicsvari et al., 1999; Louie and Wilson, 2001). For the purposes of discussion, this value will be referred to as the theta index. The theta harmonic (TH) index was computed as the sum of power in the $14-18 \mathrm{~Hz}$ band/power in the delta band.

Cluster cutting and single-unit classification. The cluster-cutting technique (McNaughton et al., 1983b) was used to separate individual single units from each tetrode, using in-house software (XCLUST; M. A. Wilson). After cluster cutting, isolated single units were classified as either pyramidal cells or interneurons, consistent with the scheme proposed by Ranck (1973). A cell was considered to be a pyramidal cell if it had the following: (1) an interspike interval histogram characteristic of hippocampal pyramidal cells, (2) been recorded on the same tetrode with other complex spike cells in the CA1 layer, (3) a spike width of at least 300 $\mu$ s, and (4) a mean firing rate $<3 \mathrm{~Hz}$ during movement. Cells were recorded during behavior and during 20-30 min rest periods flanking the behavior sessions. Only those pyramidal cells that were stable across all three epochs were included in the analyses.

Place field comparisons. Spike-raster plots and occupancy normalized firing-rate histograms were created for all pyramidal cells firing at least 40 spikes during any of the movement conditions. Information per spike (expressed in bits) was used as the quantitative measure of place specificity according to the equation provided by Skaggs et al. (1993):

$$
\text { Information }=\sum_{i=1}^{N} p_{i} \frac{\lambda_{i}}{\lambda} \log _{2} \frac{\lambda_{i}}{\lambda},
$$

where $N$ is the number of spatial bins in the environment, $p_{i}$ is the probability of occupancy in each spatial bin $i, \lambda_{i}$ is the mean firing rate for bin $i$, and $\lambda$ is the overall mean firing rate. $N$ was chosen to be 40 bins. The occupancy term, $p_{i}$, corrects for unequal sampling across the environment.

Depth of modulation. A depth of modulation index (DOM) was computed for all single units by assigning a theta phase to each spike and then creating a histogram of spike phases. From this histogram, the DOM was computed as follows:

$$
\mathrm{DOM}=\frac{\max (n \text { spikes })-\min (n \text { spikes })}{\max (n \text { spikes })},
$$

where $n$ spikes is the histogram of spike phases, max (nspikes) is the maximum, and min (nspikes) is the minimum non-zero value of the histogram. A DOM near 1 indicates strong modulation by the theta rhythm, whereas a value near 0 is indicative of a weak modulation.

Determination of the theta phase for each spike proceeded as follows. The hippocampal EEG was digitally filtered with a 6-10 Hz Chebyshev bandpass filter, and the peaks were identified based on local maxima and minima. The spike phase was then determined by subtracting the spike time from the nearest preceding theta peak and dividing that number by the total time between peaks, thus yielding a number between 0 and 1 . Average normalized depth of modulation histograms were computed for the population of active pyramidal cells from each recording session.

Single-unit autocorrelograms. The autocorrelation functions were calculated using the Matlab XCORR function. Fifty time lags of $0.02 \mathrm{~s}$ duration were chosen to reveal oscillations in the $0-25 \mathrm{~Hz}$ band. Each individual autocorrelogram was normalized by dividing the autocorrelation function by its value at zero lag. The autocorrelograms were then averaged across the population of single units within each dataset by summing each time lag bin across the entire population and dividing by the total number of single units in the population. Separate mean session autocorrelograms were made for interneurons and pyramidal cells.
Phase precession. To quantify the slope of the phase precession, the 10 theta cycles before and after the theta cycle at which the rat passed the center of the place field (defined by the occupancy normalized maximum firing rate) on each lap were identified. Histograms of the spike phases within each cycle were computed ( 40 bins/theta cycle). The mean spike phase was computed for each theta cycle. This value was computed as a function of cycle number and a line fitted by the method of least squares. Only theta cycles with at least two spikes were used. The slope and intercept of the fitted line was used for statistical comparison.

Population vector analysis. The spatial firing rate distributions for each cell in the CAR, WALK, and WORLD conditions were entered into an $N$ cells $\times 128$ bin matrix $(3.2 \mathrm{~cm} / \mathrm{bin})$. (For the CAR and WALK conditions, within-session data acquired on the RTA were used.) The columns of this matrix are estimates of the set of local population vectors. The overlap matrix for population vectors was computed (normalized dot products). For visualization purposes, the matrix values were plotted in Figure 9, using a color scale (red, overlap 1.0; blue, overlap 0 ). The width of the band along the principal diagonal of the population vector overlap matrix provides a visual indication of the scale at which the population vector changes as a function of position on the track (measured here in degrees on the circular track, in which $1^{\circ}=1.13 \mathrm{~cm}$ ). The average overlap between population vectors was then computed as a function of their spatial separation to quantify the scale of the spatial representations. Because hippocampal place fields are approximately Gaussian, this func-
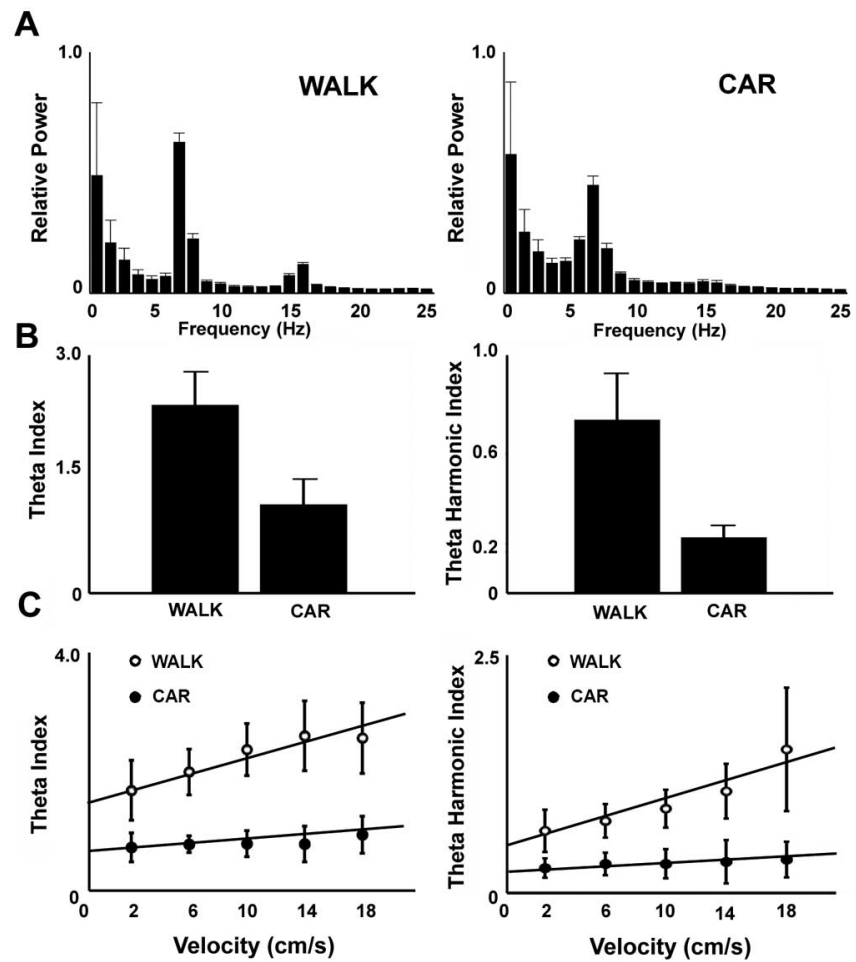

Figure 1. Effects of ambulatory self-motion on the hippocampal theta rhythm and its first harmonic $(\boldsymbol{A}-\boldsymbol{C})$. A, Representative power spectra during WALK and CAR self-motion conditions. Data are from a single recording session during which the rat drove and walked within the same session. The hippocampal theta rhythm and its first harmonic were substantially reduced during the CAR condition compared with the WALK condition. The example is representative of the results obtained in all nine within-session datasets from four animals. $\boldsymbol{B}$, The theta and $\mathrm{TH}$ indices during WALK and CAR confirmed these observations. Indices were computed by normalizing the total power in the theta $(7-9 \mathrm{~Hz})$ and $\mathrm{TH}(14-18 \mathrm{~Hz})$ by the power in the delta band $(1-4 \mathrm{~Hz})$. The two indices were significantly reduced during the CAR condition compared with the WALK condition. The mean theta and TH indices for CAR and WORLD conditions were not different. $\boldsymbol{C}$, The speed dependence of the theta and TH indices were reduced in slope in the CAR condition compared with WALK. Open and filled circles represent mean index values for WALK and CAR, respectively. Error bars represent the SEM. Data were pooled from nine recording sessions, during which the subjects engaged in blocks of CAR and WALK within the same recording session. 
tion is also approximately Gaussian, at least over its initial range. In principal, the point at which the average overlap falls to 0.5 provides a convenient, unbiased definition of the scale at which changes in spatial location are reflected in changes in the hippocampal ensemble activity; however, this function can be distorted by sampling noise and by the periodic boundary conditions imposed by the track configuration (see below and Fig. 11).

Numerical simulations. Because it was found that changes in neuronal population activity among WALK, CAR, and WORLD conditions were complex, some simple numerical simulations were performed that give some insight into some of the factors leading to this complexity. The general explanation proposed below (see Discussion) is that place field size is set by a self-motion signal whose gain is reduced successively in the CAR and WORLD conditions, resulting in the hippocampus behaving as though the rat were moving successively more slowly and on successively smaller tracks, such that the proportion of the track covered by a given field successively increased, and the proportion of highly active cells decreased. The simulations were intended to illustrate the effects of variation in the radius of the track on which the rat ran, under the assumption that absolute place field size is invariant over changes in track size, which we have verified experimentally (Maurer et al., 2005). The effects of sampling noise were also simulated to illustrate why the experimental results cannot be readily explained by the field merely becoming noisy or expanding.

The centers of 100, unit height, Gaussian place fields were distributed randomly within a unit-square environment. The place field size was defined as the SD of the Gaussian and was set to 0.1 to match the empirically derived value obtained for the WALK condition. The probability of a spike occurring at a point in space over a single time step was made
SLOW

$(4-9 \mathrm{~cm} / \mathrm{s})$

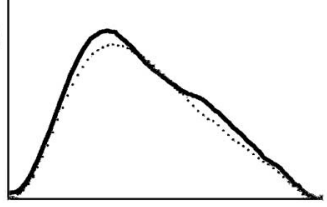

MEDIUM $(10-15 \mathrm{~cm} / \mathrm{s})$

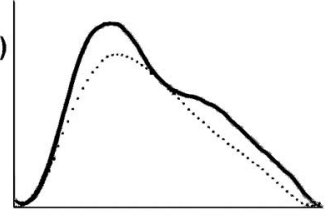

FAST $(16-21 \mathrm{~cm} / \mathrm{s})$

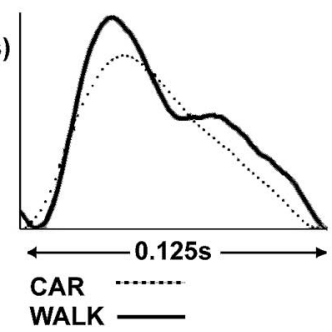

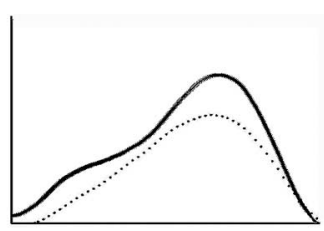
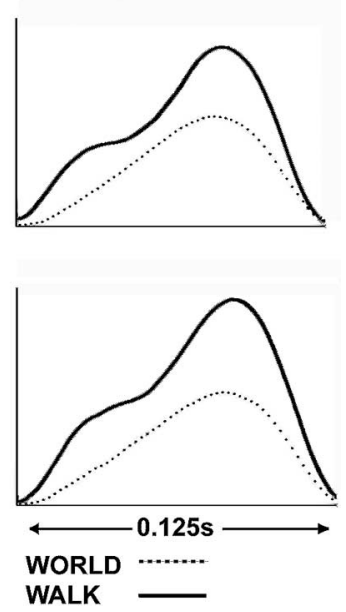

Figure 2. Influences of ambulatory self-motion on the shape of hippocampal theta waves. The three plots in each column correspond to a single recording session, during which the animal engaged in CAR and WALK in the same session (left column) or WORLD and WALK in the same session (right column). The averaged waveform was computed by finding the peaks of all theta waves in the movement condition and sorting them by the animal's velocity at the time of the wave. The data at each time point for the $0.125 \mathrm{~s}$ (single theta cycle) surrounding the peak were then averaged. The $y$-axis of the plots in each column is scaled to the amplitude of the average theta wave at high speed. During ambulatory self-motion (WALK), a clear speeddependent change in the shape of the theta wave was evident. This effect was not present during the CAR and WORLD conditions. The averaged waveform obtained during the CAR and WORLD conditions most closely resembled the waveform obtained during low-speed ambulatory movements. The two examples shown in the left and right columns exhibit mirror image shapes, which is most likely attributable to differences in the depth of the recording electrode. proportional to the height of the Gaussian at that position. The constant of proportionality could be varied to simulate the reduction in firing rate that occurs when rats run more slowly. The $360^{\circ}$ circular trajectories of various radii were simulated within this environment using $1^{\circ}$ increments. An additional parameter simulated the reduction in sampling error that would occur as either a function of the animal running more laps or a function of an increase in firing probability, such as occurs, for example, when an animal runs faster.

For each simulation, the raw data output was an $N$ cells (100 in this case), by $T$ time steps (360 in this case) matrix $Q$ (McNaughton, 1998), the rows of which represent the firing rate time series of each neuron and the columns of which represent the population vector for a given point in time or space. The population vector overlap matrices and average overlap versus distance functions were then computed as described above.

\section{Results}

\section{Velocity during WALK, CAR, and WORLD}

Despite efforts to calibrate the velocity of the vehicle to the walking speed of the rat, the mean trajectory velocity of the CAR was $17 \%$ greater than the WALK condition, and the mean during WORLD was $21 \%$ less than the WALK condition (mean velocity, in $\mathrm{cm} / \mathrm{s}$ : WALK, $10.72+0.22$; CAR, $12.56+0.24$; WORLD, $8.43+$ $0.18)$. These values include accelerations and brief stops $(<0.5 \mathrm{~s})$.

\section{Effects of self-motion on the hippocampal EEG}

Theta rhythm in the EEG recordings was clearly visible during all movement conditions; however, the theta rhythm and its first harmonic were substantially reduced in amplitude during non-
A
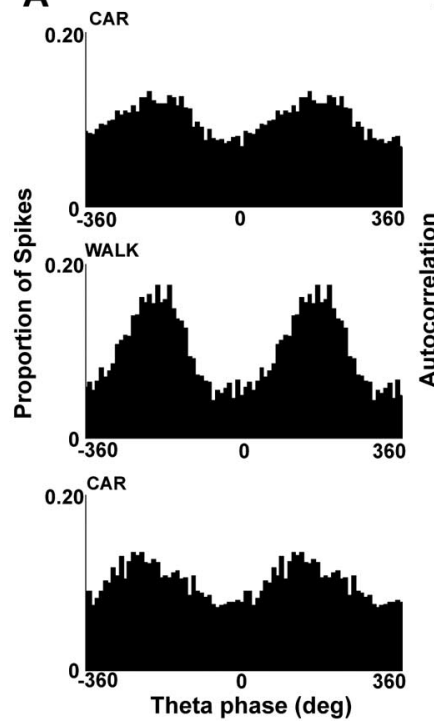

B
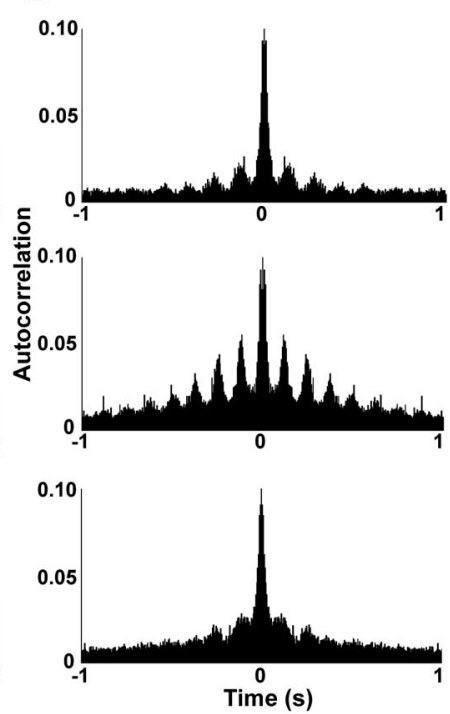

Figure 3. Effects of ambulation on the depth of theta frequency modulation $(\boldsymbol{A})$ and the "intrinsic" oscillation characteristics of CA1 pyramidal cells $(\boldsymbol{B})$. Data are from a single recording session, during which a single block of the WALK condition was interposed between two CAR driving blocks. The number of CA1 pyramidal cells used in the analysis is 25 . $A$, Modulation histograms. The firing phase (bin size, $6.3 \mathrm{~ms}$ ) for each cell in the population was computed by subtracting each spike time from the time of the nearest theta peak and dividing by the local peak-to-peak time, yielding a value between 0 and 1 (which was multiplied by 360 to convert to degrees). The total number of spikes at each phase was divided by the total number of spikes in the analysis to yield the proportion of spikes occurring at each phase of the theta cycle. These data were averaged over cells. The phase axis is double plotted to show data near the edges ( 0 and $\left.360^{\circ}\right)$. The increased theta modulation during the WALK condition returned to its reduced level during the second CAR condition. The example shown is a robust but not atypical example. $\boldsymbol{B}$, Autocorrelograms for the single units shown in column $\boldsymbol{A}$. A lag time of $0.02 \mathrm{~s}$ was chosen to reveal rhythmicity in the $0-25 \mathrm{~Hz}$ range. Notice that the intrinsic modulation frequency during CAR is $\sim 10 \%$ slower than during WALK, an effect that is related to the larger place fields and slower phase precession during this condition (see Fig. 8 and Discussion). 
ambulatory (CAR and WORLD) movements compared with walking. The power reduction in the theta and $\mathrm{TH}$ bands was consistent for all recording sessions in which there was both walking and car driving (four subjects, nine sessions). A matched-pair $t$ test comparing the within-session values of theta indices confirmed the statistical significance of the reduction (theta index: WALK, $2.66 \pm 0.42$; CAR, $1.27 \pm 0.33$; WALK $>$ CAR, $t_{(8)}=$ $4.04 ; p<0.005$; theta harmonic index: WALK, $0.87 \pm 0.23$; CAR, $0.28 \pm 0.05$; WALK $>$ CAR $\left.t_{(8)}=3.06 ; p<0.05\right)$. No statistically significant difference was obtained in the power spectra between active (rat in control) and passive (investigator in control) driving $\left(t_{(9)}=0.08 ; p \leq 0.48\right)$. Therefore, these data were pooled. These results are summarized in Figure $1 A-C$ ).

The theta and TH indices obtained during WORLD were not statistically different from those obtained during CAR (theta index: WORLD, $5.44 \pm 0.42$; CAR, $5.23 \pm 0.38$; TH index: WORLD, $0.32 \pm 0.04$; CAR, $0.33 \pm 0.03)$.

Velocity dependence of power in the theta and theta harmonic bands

The power in both the theta and $\mathrm{TH}$ bands showed an approximately linear increase with velocity during walking (Fig. 1C). The increase of power with velocity was more pronounced for the theta harmonic. The slopes of the power versus velocity relationships were greatly reduced during the non-ambulatory conditions $(n=9$; theta index: WALK, $1.27 \pm 0.36$; CAR, $0.14 \pm 0.39$; TH index: WALK, $0.54 \pm 0.23$; CAR, $0.07 \pm 0.05)$. The slopes were greater in the WALK condition for eight of nine recording
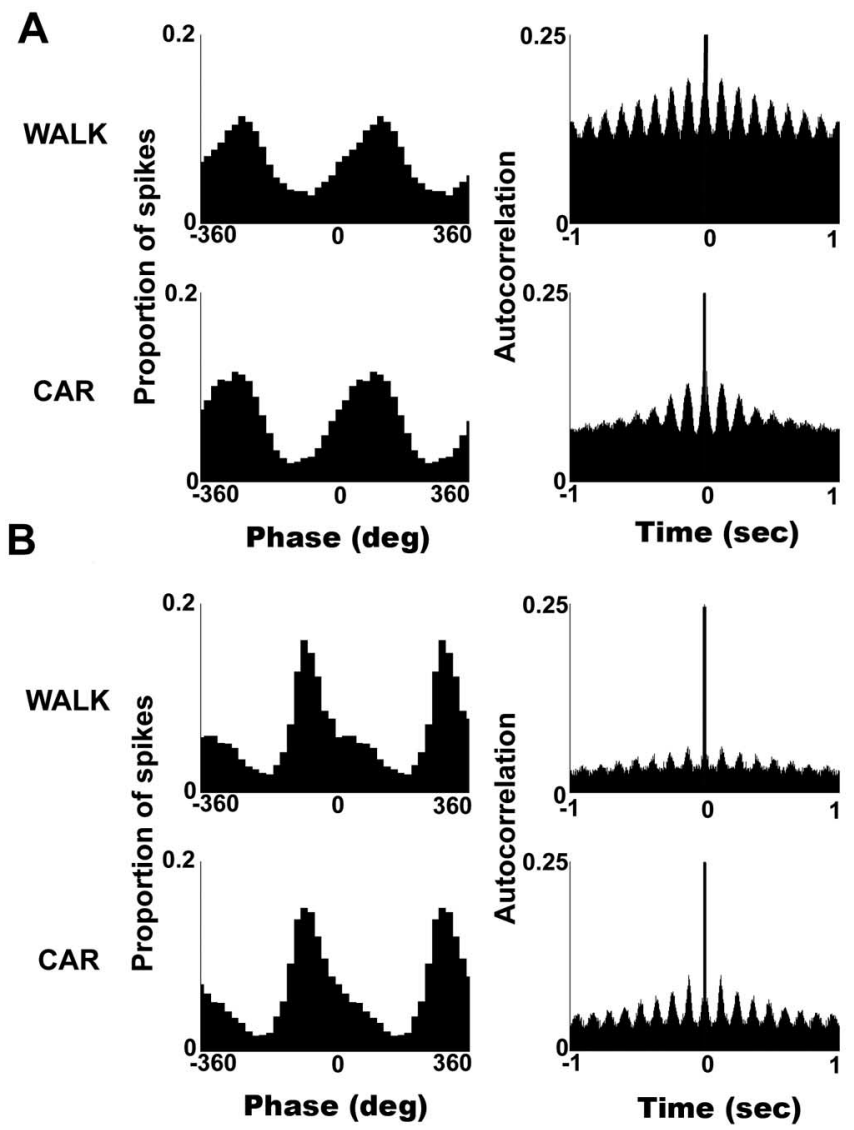

Figure 4. Effects of ambulation on the depth of modulation of CA1 interneurons $(\boldsymbol{A}, \boldsymbol{B})$. Changes were heterogeneous among the nine interneurons recorded in these experiments. Also, no effects on the phase distributions of spikes were observed.
A

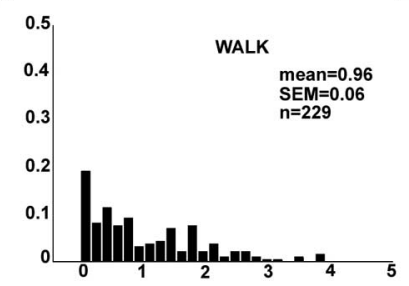

B
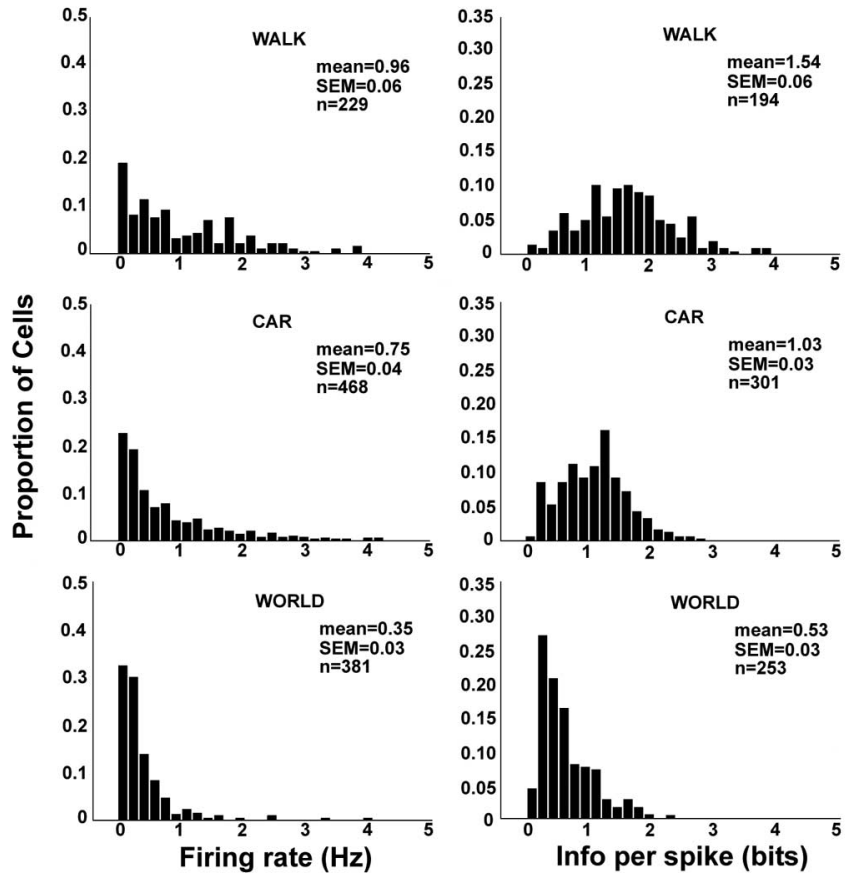

Figure 5. Effects of self-motion on the distribution of firing rates $(\boldsymbol{A})$ and spatial information per spike values ( $\boldsymbol{B}$ ) of CA1 pyramidal cells. $\boldsymbol{A}$, Firing rate distributions during WALK, CAR, and WORLD. The positive skew of the firing rate distributions during WALK is reduced during the CAR condition and further reduced during the WORLD condition. There was a significant main effect of movement condition (ANOVA; $F_{(2,1075)}=45.07 ; p \ll 0.01$ ). Only cells firing $>40$ spikes during movement were included in the information per spike distributions to obtain stable values. $\boldsymbol{B}$, The distributions of information per spike conditions during the three self-motion conditions was also less positively skewed as self-motion signals were eliminated. These changes were significant (ANOVA; $F_{(2,745)}=56.53 ; p \ll 0.01$ ). It appears from these results that optic flow, vestibular activity, and proprioception/motor efference each contributes $\sim 0.5$ bits of information to the spatial information content of hippocampal pyramidal cell discharge during normal locomotion.

sessions of the within-session RTA data for the theta index and for all nine recordings for the TH index. These differences were significant using the sign test (theta index: $p<0.04$; TH index, $p<0.004$ ). A Wilcoxon matched-pairs signed-rank test showed that the slope of $\mathrm{TH}$ versus velocity was significantly reduced $(p<0.003)$. The slope difference of the theta versus velocity relationship did not reach significance $(p<0.12)$.

The ambulatory velocity dependence of the theta and $\mathrm{TH}$ power was associated with a pronounced effect of ambulatory self-motion on the shape of theta waves (Fig. 2). A common characteristic of theta waves acquired from electrodes placed near the hippocampal fissure is the presence of "shoulders" in the averaged and select individual waveforms (Buzsáki, 1986); this shoulder appears to give rise to the harmonic peak in the power spectra. The prominence of this characteristic during walking was dependent on the ambulatory velocity of the animal. Importantly, the average theta wave obtained during non-ambulatory movements was similar to that obtained during low-velocity walking.

Effects of self-motion signals on the modulation of single-unit activity by the theta rhythm

There was a small, but statistically significant, reduction $(\sim 10 \%$ on average) in the DOM of pyramidal cell firing by the theta rhythm during non-ambulatory movements (WALK, $0.82 \pm$ 
0.02; CAR, $0.76 \pm 0.01$; group mean $t$ test, $\left.t_{(320)}=2.99 ; p<0.01\right)$. The influence of ambulation on DOM was easily seen within subjects in the modulation histograms (Fig. $3 A$ ) and the autocorrelograms (Fig. $3 B$ ). There was a heterogeneous effect of movement condition on the depth of firing rate modulation of the $(n=9)$ inhibitory interneurons (Fig. $4 A, B$ ).

\section{Effects of self-motion on place field size and firing rate}

Removal of the ambulatory self-motion signal in the CAR condition significantly reduced the information content (Fig. 5B) of active hippocampal principal cells. The additional removal of vestibular cues in the WORLD condition resulted in an additional and approximately equal reduction in information content. The information content was as follows: $1.54 \pm 0.06$, $1.03 \pm 0.03$, and $0.53 \pm 0.03$ bits for WALK (194 units), CAR (301 units), and WORLD (253 units), respectively. There was a significant main effect of movement condition (ANOVA; $F_{(2,744)}=173.33 ; p<$ $0.001)$. The reduced information content corresponded to an increase in place field size (Figs. 6, 7) and not multistability or randomization of spike position. Indeed, place fields during the CAR condition were remarkably stable over many laps ( $>100$ in many cases).

The sparsity of the distribution of firing rates was affected by movement condition (Fig. 5). The overall pattern of these effects was similar to the effects of selfmotion on the information per spike distribution, with the distribution obtained during WALK exhibiting a greater proportion of units firing in the $1-3 \mathrm{~Hz}$ range than the firing rate distribution for CAR. Also similar to the distribution of information per spike values, the distribution of firing rates was further shifted toward lower values during the WORLD condition. The means and SEMs were $0.96 \pm 0.06$, $0.75 \pm 0.04$, and $0.35 \pm 0.03 \mathrm{~Hz}(n=229,468$, and 381 for WALK, CAR, and WORLD, respectively). An ANOVA of all data pooled across apparatuses revealed a significant effect of movement condition $\left(F_{(2,1075)}=45.17 ; p<0.001\right)$. These changes in firing rate distributions correspond to a reduction in the number of cells with defined place fields in the CAR and WORLD conditions.

There also appeared to be evidence of "remapping" when the movement condition was changed. As seen in the examples in Figure 7, within a given session, the presence and/or location of a place field for a given cell frequently varied as a function of movement condition.

\section{Effects of self-motion on phase precession}

A regression analysis of position on the maze versus firing phase relative to the EEG theta rhythm was performed for the subset of cells $(n=68)$ exhibiting place selectivity in either WALK or CAR
CAR
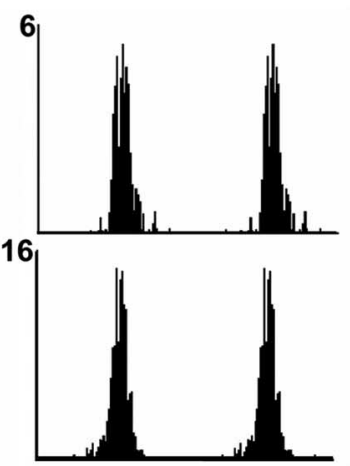

14

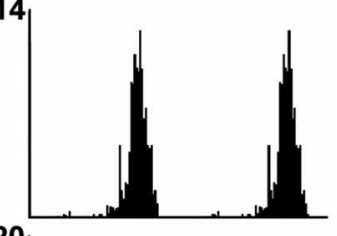

20

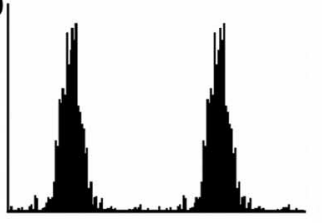

5

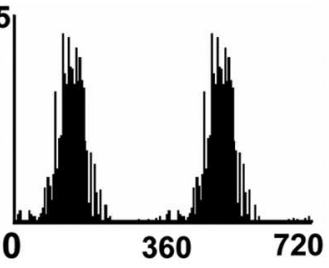

720
WORLD
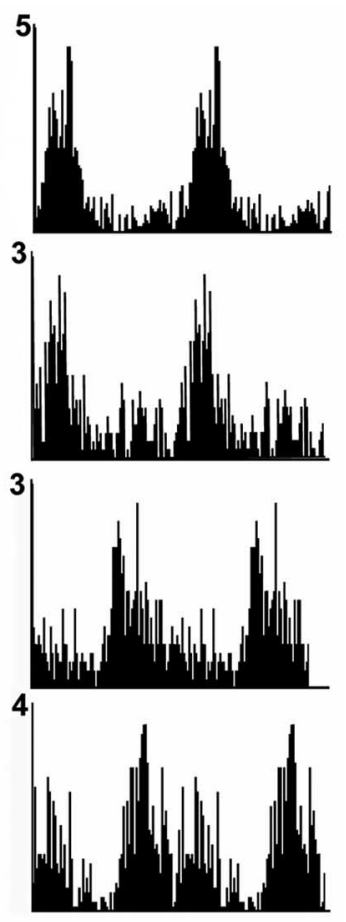

4

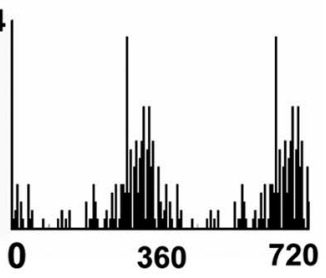

\section{Position on Track (deg)}

Figure 6. Illustration of the five "best" place fields during the WALK, CAR, and WORLD movement conditions. Each of the 15 plots is from a different cell. Data are plotted over two cycles of the position on the circular track to illustrate the shape of the fields ms of the five pyramidal cells with the highest information per spike values in each of the three self-motion condition after sorting the entire set of cells by information per spike. Notice also that even the most robust place fields in the WORLD condition were associated with low peak firing rates. The majority of cells in this condition were virtually silent.

and having adequate theta recordings. In addition, fields located near the goal locations and "double fields" were excluded from this analysis. Under both conditions, a systematic phase retardation of up to $360^{\circ}$ ["phase precession" (O’Keefe and Recce, 1993; Skaggs et al., 1996)] could be observed as the animal passed through the place field. The same effect could sometimes be seen in the WORLD condition, but these data were considerably noisier because of the greatly reduced spatial information content and firing rates. For this reason, the WORLD data were excluded from this analysis. Figure 8 shows typical examples of phase precession in WALK and CAR. There was no apparent effect of movement condition on the total range over which phase changed, but there was a considerable reduction in the spatial rate of change of phase in the CAR condition. Without an explicit model, it is difficult to quantify the parameters of phase precession because both mean phase and phase variability typically change in a nonlinear manner as a function of position (Yamaguchi et al., 2002), but a first approximation of the rate of phase change with position was calculated using a linear regression (see 
A
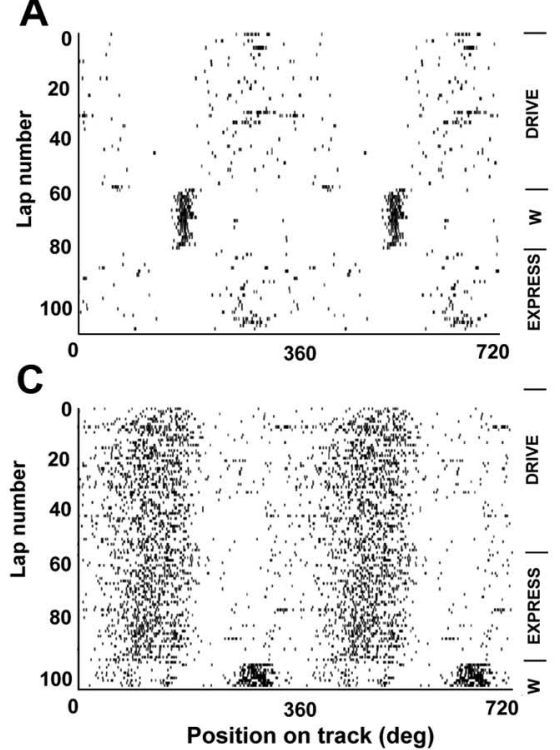

B
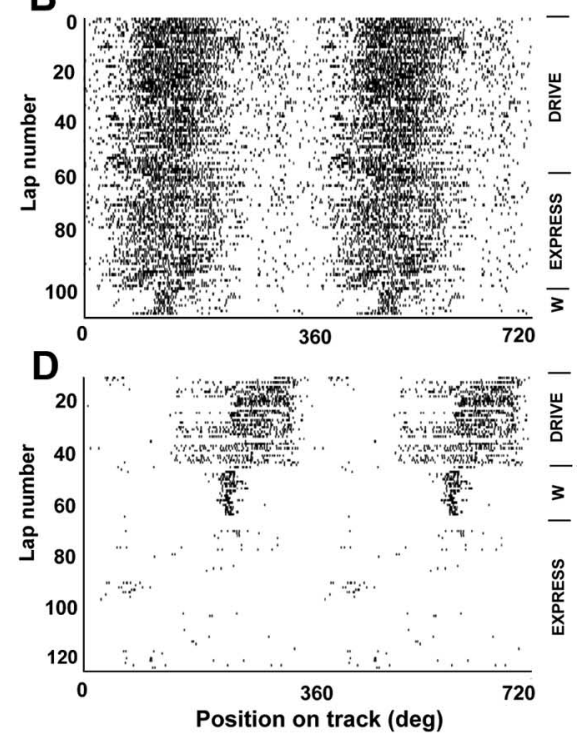

Figure 7. Example raster plots obtained during WALK (W) and CAR (DRIVE or EXPRESS) self-motion conditions (A-D). Each tick mark represents the location and lap number of a single spike recorded from a CA1 pyramidal cell while the rat moved along the circular track. Only spikes recorded while the rat was moving at a velocity $>5 \mathrm{~cm} / \mathrm{s}$ are shown. The movement condition for each block of trials is shown on the right-hand vertical axis of each plot. EXPRESS refers to a block of CAR trials during which the rat was passively driven around the track, and DRIVE refers to CAR trials during which the rat drove actively. The position axis for the circular track is double plotted to eliminate edge effects. The spikes acquired during the WALK condition occurred over a much smaller area of space than those obtained during the CAR condition. Regardless, the single units acquired during the CAR condition fired in consistent places over many laps (sometimes >100 laps, as in the examples), indicating that the place representation was not multistable or shifting from lap to lap. The data within each example are taken from the same recording session and illustrate the fact that that the presence and position of place fields for a given cell often changed with the movement context (remapping).

Materials and Methods). Consistent with the substantial increase in place field size during CAR (Figs. 5-8), the slopes of these functions were approximately threefold steeper during WALK (slope: WALK, $-0.045 \pm 0.007$; CAR, $-0.014 \pm 0.005$; ANOVA; $\left.F_{(1,66)}=10.14 ; p<0.01\right)$.

\section{Effects of self-motion on the population representation of space}

The within-session spatial firing rate distributions of active cells recorded during both WALK $(n=152)$ and CAR $(n=96)$ on the RTA were plotted in descending order of spatial information per spike to illustrate the increase in place field size during the CAR condition. Data from active cells $(n=217)$ in the WORLD condition were obtained from different subjects during trials in which the animals drove the WORLD exclusively throughout the entire recording session. The spatial firing distributions for the three conditions were aligned on their respective peaks. The results show a consistent pattern of increasingly diminished spatial tuning during CAR and WORLD compared with WALK.

The population vector overlap matrices and the average change in overlap as a function of distance on the track were computed (described in Materials and Methods). As shown in Figure 9, the scale at which the CA1 population firing rate vector changes with position was approximately three times larger during CAR than during WALK and substantially increased again during WORLD to the point that most cells that were active on the track fired over most of the track, with relatively low rates. The possible causes of these effects are explored in the numerical simulations reported below (Figs. 10, 11).

\section{Numerical simulations}

As will be elaborated in Discussion, the differences between the WALK, CAR, and WORLD conditions are consistent with what would be observed if, during CAR and WORLD, the rats were moving on a circle of smaller radius and at slower speed, and the place field size was expressed in terms of angular position. In other words, in the CAR condition, the hippocampus appears to behave as if its head-direction input continues to provide accurate information about head direction, but the gain of the signal that provides linear speed information is reduced. As a result, the hippocampus behaves as if the rat was completing full circuits in the same lap time but moving more slowly; hence, the radius of the track must appear smaller. Place fields would thus cover more of the track, but fewer cells would have fields on the track. The main purpose of the numerical simulations was to gain insight into the factors leading to the initially unexpected results shown in Figure 9 in which the mean population vector overlap versus distance function for the WORLD condition did not match the naive prediction that it would resemble those for WALK and CAR but with a more gradual decay rate. With this question in mind, the parameters were adjusted to approximate the observed functions (Fig. 10). The three variable parameters used in the main simulations for the WALK, CAR, and WORLD conditions were the radius of the track $(0.45,0.15$, and 0.05 , respectively), the signal-to-noise parameter $(45,45$, and 2 , respectively), which is effectively proportional to the number of laps over which the fields were averaged, and the peak within-field firing probability, which is known to increase linearly with running speed (Ekstrom et al., 2001). Both the number of laps and the peak firing rates affect the signal-tonoise ratio of the estimate of place field parameters. In the WORLD condition, the relatively low signal-to-noise parameter reflects the fact that there were both many fewer laps and much lower firing probabilities. In addition, simulations were run to contrast the effects on firing rate distributions of reduced track size, keeping place field size constant, versus a constant track size with increasing place field size.

Three important principles emerged. First, although the overlap is, by definition, 1.0 for a given vector with itself, the overlap function drops abruptly at the next interval, before beginning a gradual, approximately Gaussian decline. The magnitude of this drop is inversely related to the signal-to-noise ratio of the recorded place fields. In the limit of a very large number of trials, or very high local firing rates, the initial drop becomes negligible, but it is not necessarily negligible otherwise. The steep initial drop in the WORLD condition is thus attributable to the greatly reduced signal-to-noise of the spatial firing rate distributions under this condition, which is commensurate with the greatly reduced mean firing rates and much smaller number of laps than in the CAR condition. The lack of a large initial drop in the CAR condition, despite the reduced firing rates, can be attributed to the 


\section{WALK}

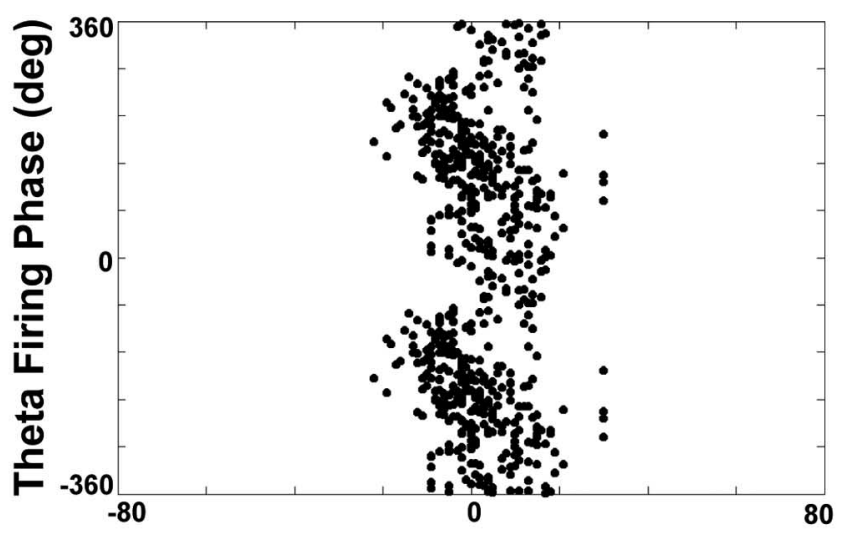

CAR

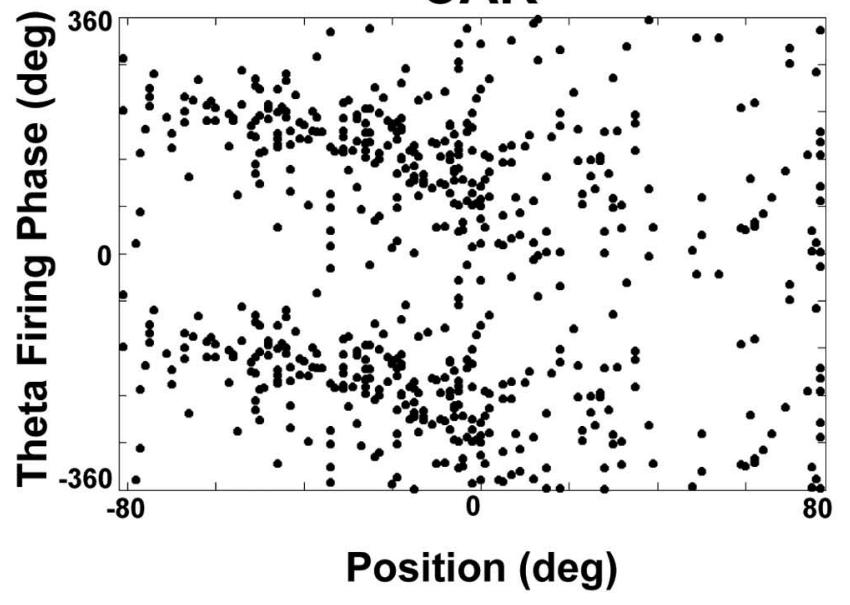

Figure 8. Reduced slope of phase precession during non-ambulatory movement. Each plot represents the phase-by-position firing characteristic of a typical single unit with a place field recorded during CAR and WALK movement conditions. Each of the black dots represents the animal's position and the corresponding firing phase for a single spike. The $y$-axis represents firing phase and is double plotted to reveal relationships near the phase edges. The $x$-axis is the position of the animal during each spike and is centered on the centroid of the spatial firing. During WALK, the slope of phase shift versus position was approximately three times greater than during CAR, but the range of phase shift was not different.

fact that the number of laps was approximately four times greater in this condition than in the WALK or WORLD conditions.

The second point of note is that the population vector overlap versus distance function, for Gaussian place fields, only reaches an asymptote of zero in the limit of an infinite linear track. The periodic boundary condition, attributable to movement in a circular trajectory, means that the minimal value of the function is reached at $180^{\circ}$, after which the function increases again. The smaller the track size, holding field-size constant (i.e., the larger the place field relative to the track), the larger this minimum value is (Fig. 11). These considerations provide a plausible explanation for the shapes of the observed overlap decay functions; however, the same general effects are observed if place fields are increased, while holding track-size constant, again increasing the size of the field relative to the track. The effects on firing rate distributions, however, are radically different under these two conditions. In the constant-field-size, variable-track-size condition, the mean firing rates do not change significantly unless firing rate is adjusted to match simulated running speed when the lap time is held constant, but the variance of the firing rate dis- tributions increases dramatically (i.e., the distributions become sparser, with many more very-low-rate cells and a few muchhigher-rate ones). In the variable-field-size, constant-track-size condition, the mean firing rates increase steeply with field size, and the variance is reduced (the distributions become much less sparse). Thus, the simulations strongly favor the constant-fieldsize, variable-track-size and speed parameter hypothesis. The result of two simulations illustrating the contrasting effects of changing track size (and running speed) versus changing place field size on mean firing rate and the sparsity of the firing rate distributions are given in Table 1.

\section{Discussion}

Successive restriction of ambulatory and vestibular self-motion cues each reduced the total spatial information per spike of place cells by $\sim 0.5$ bits, leaving $\sim 0.5$ bits when only visual motion signals remained. In contrast, place fields often "remapped" between movement conditions, implying that the changing relationship of the animal to landmarks played little role in updating the hippocampal activity pattern. These data are consistent with theoretical models (McNaughton et al., 1996; Touretzky and Redish, 1996; Redish and Touretzky, 1997; Samsonovich and McNaughton, 1997) and empirical studies (Markus et al., 1994; Gothard et al., 1996), indicating that the hippocampus either performs path integration or is strongly driven by path integration signals and further support the conclusion that the scale at which hippocampal activity changes with location is primarily based on self-motion signals.

Vestibular and optic-flow cues may have contributed in two ways. The head-direction cell system (Taube et al., 1990a,b; Taube, 1995) performs a neural integration of head angular velocity to produce a signal related to head direction (McNaughton et al., 1991; Blair and Sharp, 1995; Skaggs et al., 1995; Zhang, 1996). Head direction can strongly influence which pyramidal cells fire at a given location (McNaughton et al., 1983a). Thus, the residual spatial variation in firing in the CAR and WORLD conditions may reflect either changes in the head-direction cell input or a direct effect on hippocampal neurons of the linear and angular velocity information conveyed by vestibular and optic-flow signals. For example, electrical stimulation of the medial vestibular nucleus increases CA1 firing rate (Horii et al., 2004).

The theta rhythm in CAR and WORLD conditions behaved as if the rat's speed of movement were actually reduced; however, the largest effect on theta occurred with the elimination of ambulatory signals. There was little additional effect when vestibular signals were also eliminated. This may reflect statistical error or may indicate nonlinearities in the manner in which the effects of movement signals on theta power summate.

The firing of active pyramidal cells oscillates at a frequency somewhat faster than the local field potential, leading to the phase precession effect (O'Keefe and Recce, 1993; Skaggs et al., 1996). Phase precession occurred in the absence of locomotion, and a corresponding subtle slowing of the intrinsic oscillation frequency during CAR driving can be observed in Figure 3. (The term "intrinsic oscillation frequency" is not meant to imply any specific membrane dynamics or mechanism, only that the firing rate fluctuates with a period that differs from the local field.) This may have bearing on the mechanism of the increased place field size. Over a place field, firing phase shifts by $\sim 360^{\circ}$ but never more, which provides an unbiased operational definition of a place field (McNaughton et al., 1997). Cells frequently exhibit more than one place field in any given environment (Barnes et al., 1983), and multiple place fields are associated with multiple cy- 
Place Fields
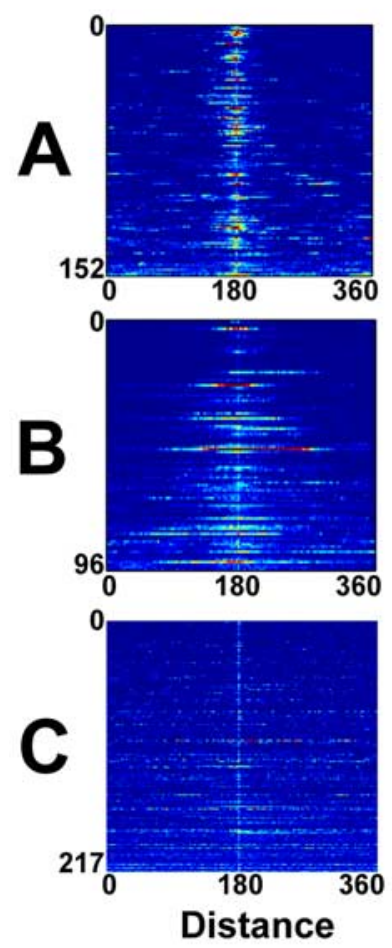

Overlap Matrix
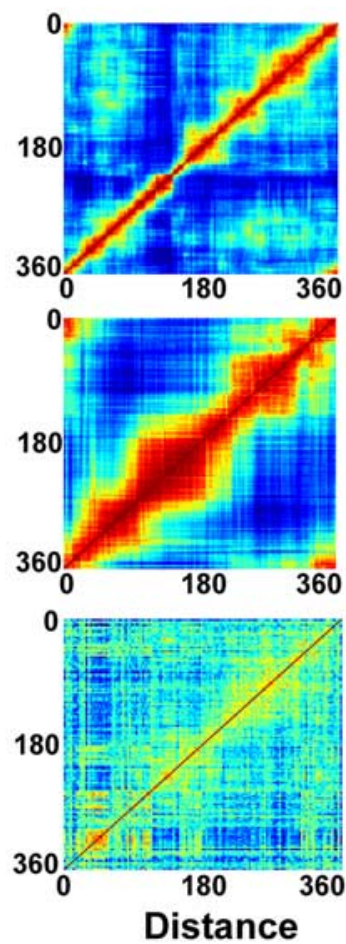

Overlap vs. Distance
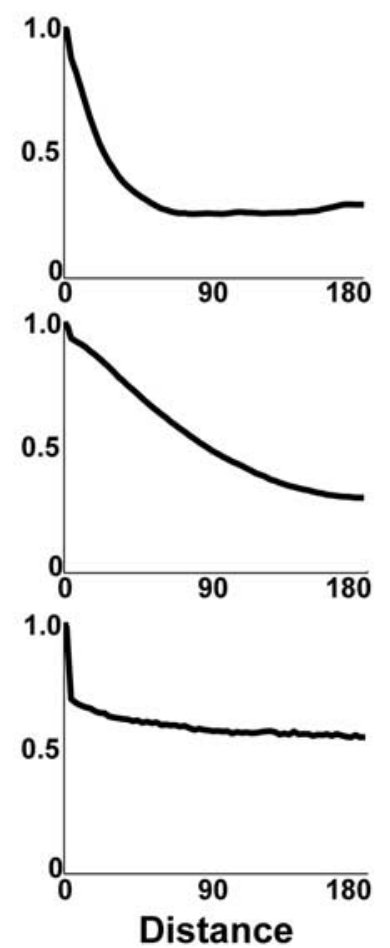

Figure 9. Effects of ambulatory self-motion on the scale of the hippocampal spatial metric. The spatial firing rate distributions for all active (firing $>40$ spikes) pyramidal cells recorded in both WALK $(\boldsymbol{A} ; n=152)$, CAR $(\boldsymbol{B} ; n=96)$ conditions and for those recorded separately in the WORLD $(\boldsymbol{C} ; n=217)$ condition were arranged in descending order of spatial information per spike. The distributions were then aligned on their respective peaks and are illustrated in the first column (Place Fields). The area of space represented by the firing of the pyramidal cells during the CAR condition is approximately three times larger than that represented during WALK. In the WORLD condition, approximately two-thirds of the cells fired only rarely and most others fired over most of the track. Composite population vectors were constructed from the entire sample of recorded cells for each condition and position. The overlap matrices (normalized dot product) for these composite vectors are illustrated in the second column (red, overlap 1.0; blue, overlap 0). The width of the diagonal stripe provides an indication of the scale at which the population vector changes as a function of position on the track (measured here in degrees on the circular track, in which $1^{\circ}=1.13 \mathrm{~cm}$ ). The lack of a prominent diagonal stripe in the WORLD condition results from three factors. First, many of the active cells fired over nearly the whole track. Second, there were many fewer laps for the WORLD condition than for the CAR condition, resulting in poorer signal-to-noise of the place fields. Third, the overall firing rates were substantially lower, again contributing to reduced signal-to-noise. These effects are illustrated in the numerical simulations shown in Figures 10 and 11. The average rates at which the overlap between successive population vectors changed as a function of distance are illustrated in the third column.

cles of phase precession. By the phase-shift-based definition, two place fields from the same cell can overlap, in which case, doublets of firing occur at distinct points in each theta cycle (McNaughton et al., 1997). Moreover, place fields exhibit experience and NMDA receptor-dependent expansion during repeated route following (Mehta et al., 1997; Ekstrom et al., 2001). When fields expand, the range of the phase shift does not change, but the rate of change of phase with position is slowed. This implies a slowing of the intrinsic oscillation frequency of the individual cells.

One model for phase precession (Jensen and Lisman, 1996; Tsodyks et al., 1996) suggests that it results from asymmetry in the synaptic matrix, leading to a phenomenon whereby the initial firing within the place field occurs late in the theta cycle and is driven by intrinsic connections, whereas the firing as the animal leaves the field occurs early in the cycle and is driven by the external input. According to this hypothesis, the hippocampus performs a look-ahead function within each theta cycle, which implies that the range of the look-ahead increases in the CAR and
WORLD conditions. It is possible that this could arise from reduced inhibitory modulation and/or a change in the relative weights of the intrinsic and extrinsic excitatory inputs. Wallenstein and Hasselmo (1997) suggested that $\mathrm{GABA}_{\mathrm{B}}$ receptor activation may selectively reduce the relative weight of intrinsic connections. Periodic activation would allow the network to be driven more by the extrinsic inputs during the early portion of the theta cycle and more by the intrinsic connections later in the cycle. A reduction in $\mathrm{GABA}_{\mathrm{B}}$ receptor activation during CAR and WORLD conditions might, therefore, lead to an accelerated "look-ahead" function and hence larger fields.

A place field may be characterized as a rhythmic burst of firing that begins late in a theta cycle and terminates after the phase has shifted earlier by one complete cycle. The number of cycles required for this process is inversely related to the increase in intrinsic oscillation frequency of the cell above the theta frequency of the field potential. This frequency increases as running speed increases (as does firing rate per se) (McNaughton et al., 1983a), such that the number of theta cycles required to span the field decreases but the size of the field remains constant. At constant running speed, even small changes of the intrinsic frequency can result in substantial changes in the number of cycles required for a $360^{\circ}$ phase shift and, hence, the apparent size of the place field.

When rats run on a running wheel (thereby eliminating optic-flow and vestibular cues), there is a linear increase in firing rate with running speed for pyramidal cells that are active in the wheel, and an increase in theta and TH power, similar to that observed in the WALK condition (Czurko et al., 1999). In contrast, ambulatory signals, in the absence of changes in location, typically resulted in sustained firing of "wheel cells," with no updating of the hippocampal code over time and no phase shift. Thus, locomotion per se, in the absence of movement, is not typically sufficient to update the hippocampal firing pattern. Locomotion on a linear track in total darkness (Gothard et al., 2001), however, causes updating of the code relative to a variably located start box (i.e., not relative to any fixed cues). Thus, hippocampal updating, when the animal is actually moving, may depend on the integration of acceleration signals and the corresponding perception of true motion in space. The lack of an effect of ambulatory signals on hippocampal updating, in the absence of either vestibular or optic-flow signals, may indicate some gating mechanism that determines whether ambulation signals are included in the overall estimate of movement speed. Interestingly, G. Buzsáki (personal communication) indicates that, in some of the running-wheel experiments not reported by Czurko et al. (1999), the recorded neuron suddenly began to shift phases as though the animal were moving slowly in space. One can imagine the possibility that a 


\section{Track}
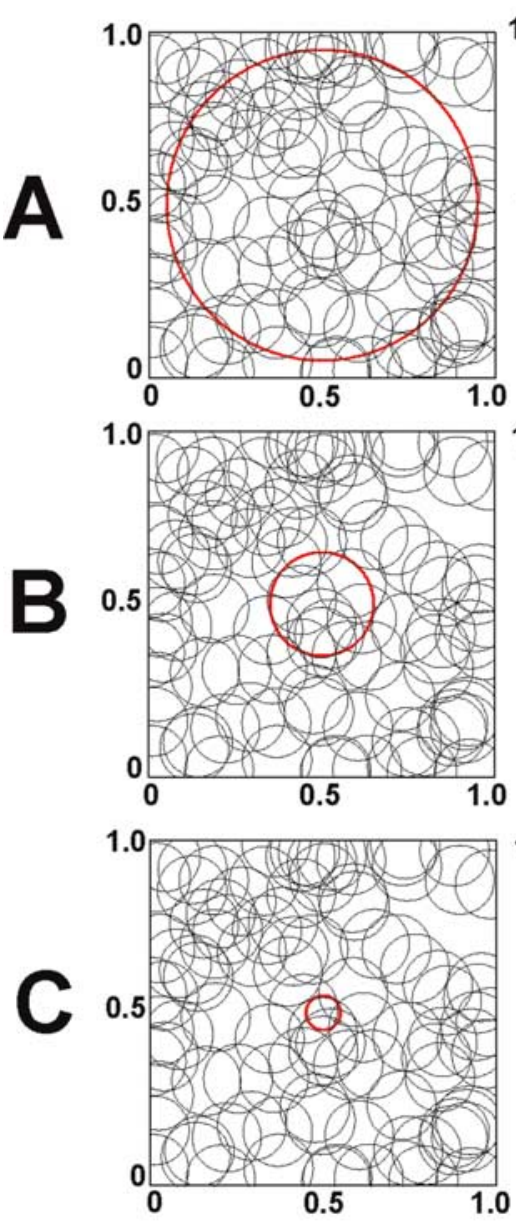

\section{Linear Distance}

Place Fields
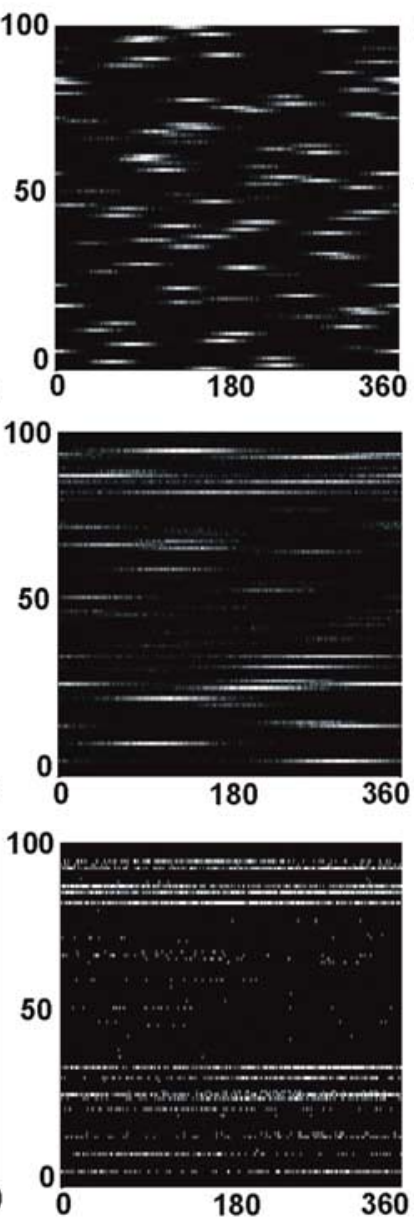

Angular Distance
Overlap Matrix
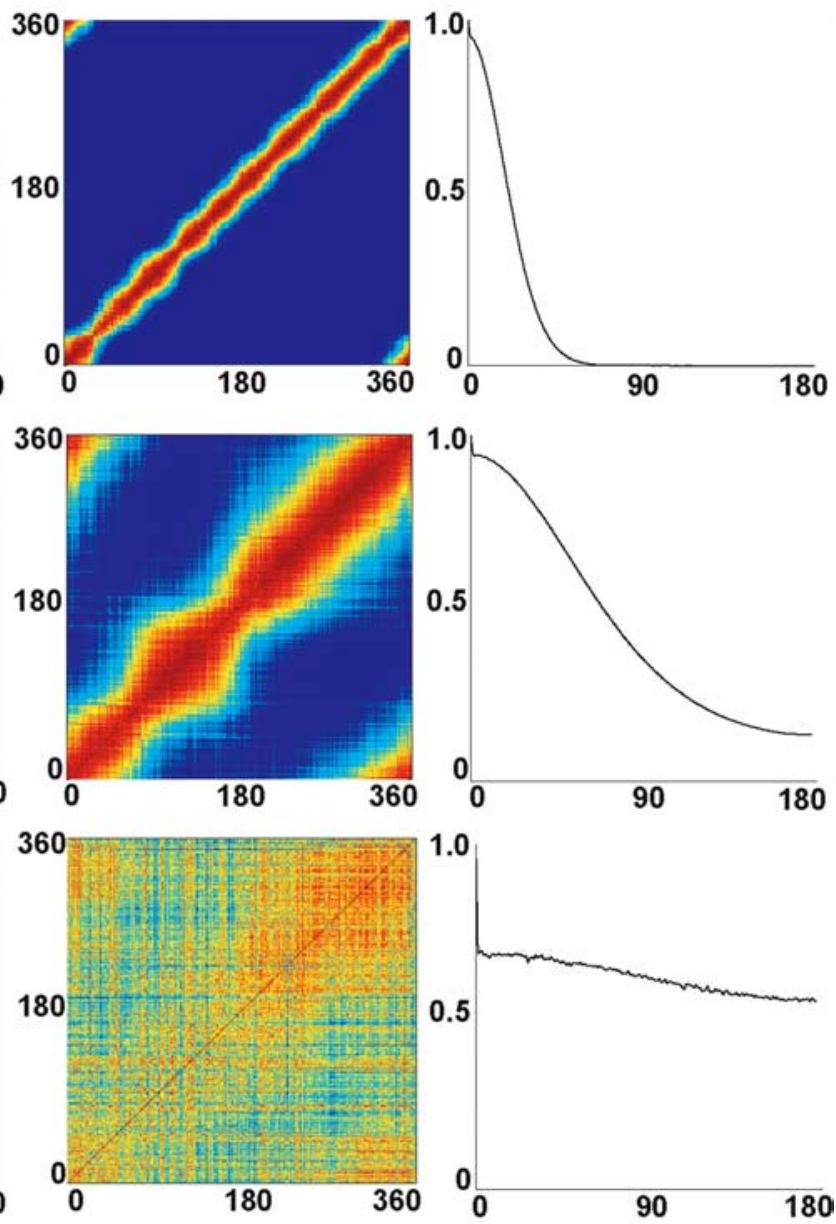

Angular Distance
Overlap

vs.

Distance

Figure 10. Numerical simulations of how a reduction in the gain of a self-motion signal would be expected to affect relative place field size and population vector relationships if the hippocampal population vector was updated as a function of distance moved (path integration) rather than changes in external inputs. Because the head-direction signal is still operative and the lap time remains constant, reducing the linear movement signal would be equivalent to traversing a smaller circle at lower speed. The result is that fewer place fields are traversed, but those that are traversed cover a larger portion of the track. Place fields were simulated as Gaussian firing probability distributions (1 per cell), with centers distributed randomly in a unit square. The black circles in the first column represent the SD of the Gaussians, and the red circles represent the circular trajectories of the rat on the track. Simulated place fields are shown in the second column in which firing rate is represented as a gray scale value (black, 0 ). The vertical axis represents cell number (1:100), and the horizontal axis represents the angular coordinate (degrees). Variations in net signal-to-noise ratio (see Fig. 9) were simulated by varying the number of sampled laps, although in reality, this parameter is also affected by changes in firing probability. Notice that, as the circle diameter is reduced progressively $(0.45,0.15$, and 0.05 for $\boldsymbol{A}-\boldsymbol{C}$, respectively), progressively fewer cells exhibit activity on the circle, but those that do exhibit activity fire over progressively larger regions of the track. The corresponding population vector overlap matrices and mean overlap versus distance (expressed in angular coordinates) functions are shown in the third and fourth columns, respectively.

shift in the animal's reference frame from the room to the wheel itself might trigger such an event. Humans can estimate passive linear displacements and reproduce translational velocity profiles using only inertial otolithic signals (Israel and Berthoz, 1989; Israel et al., 1993; Berthoz et al., 1999), and damage to the vestibular apparatus profoundly disrupts spatial learning and place cell firing in rodents (Stackman and Taube, 1997; Stackman et al., 2002).

An alternative to the hypothesis that a reduction in the gain of a self-motion signal was responsible for the CAR and WORLD effects might be that place fields simply expanded. This might occur, for example, if they were driven by approximately Gaussian-distributed external inputs with inhibitory threshold- ing, and inhibition was simply reduced. As shown by the simulations, this explanation predicts an increase in the mean and a decrease in the sparsity of the firing rate distributions, whereas the opposite occurred. Thus, the CAR and WORLD condition activity is what would be observed if the rat were physically moving at a slower speed around a smaller track (constant lap time), and the place fields were plotted in angular rather than linear coordinates.

The experimental challenges of training rats to drive coupled with the exploratory nature of these experiments did not allow for recording sessions in which the animal engaged in all three movement conditions within the same recording sessions; therefore, the appropriate caveats should be considered. It was clear, 


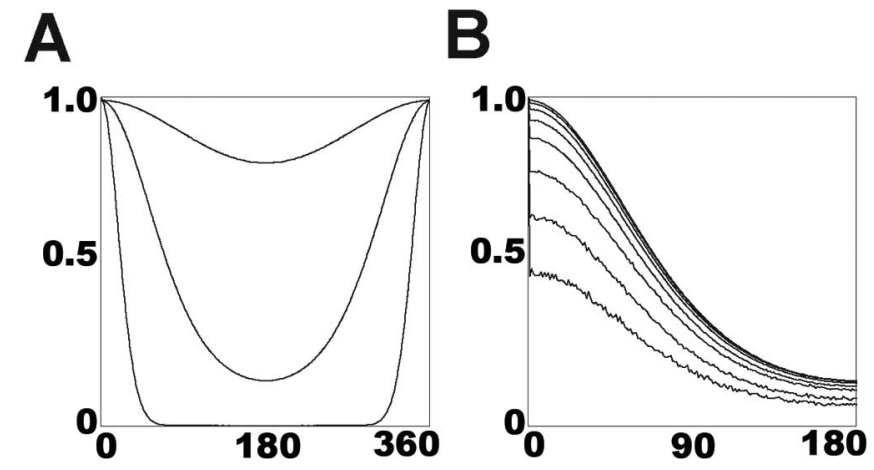

Figure 11. $A$, Effects of the periodic boundary condition on the population vector overlap versus angular position function as the track size is varied (same size parameters as in Fig. 10). In these simulations, the signal-to-noise parameter was maximized, so that all curves begin and end at 1.0, with no abrupt drop at the first interval. Note that as the circle size is reduced (i.e., relative place field size increases, the functions never go to zero. $\boldsymbol{B}$, Simulations for the intermediate sized track in Figure 10, with the signal-to-noise parameter (number of laps) successively doubling from 1 (lowest curve) to 128 (highest curve).

\begin{tabular}{|c|c|c|c|}
\hline & WALK & CAR & WORLD \\
\hline \multicolumn{4}{|c|}{ Variable track } \\
\hline Rad & 0.45 & 0.15 & 0.05 \\
\hline$S D$ & 0.10 & 0.10 & 0.10 \\
\hline Mean & 1 & $1(0.02)$ & $1(0.006)$ \\
\hline Sparsity & 0.763 & 0.223 & 0.118 \\
\hline \multicolumn{4}{|c|}{ Variable place fields } \\
\hline Rad & 0.45 & 0.45 & 0.45 \\
\hline SD & 0.10 & 0.20 & 0.30 \\
\hline Mean & 1 & 3 & 5.4 \\
\hline Sparsity & 0.763 & 0.966 & 0.983 \\
\hline
\end{tabular}

The place field size parameter (SD) and the track size parameter (Rad) for the variable-track size condition are as used for the simulations in Figure 10. Mean rates are shown relative to the putative WALK condition. The numbers in parentheses in the variable-track condition represent the relative rates, assuming that firing probability scales linearly with simulated running speed, if the lap time was held constant and the track diameter varied. Thus, under the hypothesis of a loss of gain in the speed parameter, firing rates decrease and the firing rate distribution becomes more sparse (smaller sparsity index), which is what is actually observed in the data. Under the hypothesis that all place fields simply get bigger, the mean rates increase substantially, and the firing rate distributions become less sparse (larger sparsity index).

however, that the scale of the place representation was altered by the stepwise loss of self-motion information. These effects were shown either within sessions, in the case of most of the WALK versus CAR comparisons, or within subjects for most of the CAR versus WORLD comparisons.

A second caveat concerns whether the changes in neurophysiological measures obtained with complete removal of a selfmotion input signal provides a good measure of the influence of that signal under normal conditions. Moreover, in the case of the WORLD condition, the reduced spatial information resulting from the combined removal of ambulatory and vestibular selfmotion may have been different if it were somehow possible to remove only the vestibular self-motion signal. Previous experiments (Czurko et al., 1999) showed that the velocity dependence of theta and its first harmonic is not dependent on optic-flow or vestibular self-motion information. Thus, in a condition in which only vestibular self-motion signals were eliminated, the velocity dependence provided by the ambulatory system would presumably remain intact. Lesions of the vestibular system (Stackman et al., 2002) and head-directional system (Calton et al., 2003) reduce, but do not completely abolish, CA1 spatial information content. It remains to be seen whether it is the loss of information or the conflict between self-motion signals that is driving these results. Future experiments should examine whether manipulations of the relative gain of each self-motion signal can change the results in accordance with the model presented here.

In summary, the spatial scale over which CA1 pyramidal cells change their activity pattern is determined primarily by selfmotion signals derived from a possibly complex interaction of motor commands, proprioceptive feedback, optic flow, and vestibular information. Although the hippocampal activity can be altered radically by either external inputs or internal state variables such as behavioral context or the relationship between the head-direction system and external cues, it is unlikely that specific environmental features or the spatial relationships of these features relative to each other or to the observer are the primary determinants of place field size and distribution. Rather, it appears more likely that the initial allocation of place fields is determined primarily by the immediately preceding hippocampal state and the distance and direction moved by the animal. A model for the synaptic matrix that might underlie such behavior, as well as how external inputs become associatively bound to the current "map" and hence enable reference frame shifts within it, was proposed by Samsonovich and McNaughton (1997). Such dynamics are perhaps not surprising in a system so far removed synaptically from the periphery, so strongly modulated by movement and directional parameters, and in which the majority of inputs to a given pyramidal cell come from other hippocampal pyramidal cells. Nevertheless, the mechanism by which movement controls the updating of the hippocampal spatial code remains to be discovered.

\section{References}

Barnes CA, McNaughton BL, O'Keefe J (1983) Loss of place specificity in hippocampal complex spike cells of senescent rat. Neurobiol Aging 4:113-119.

Berthoz A, Amorim MA, Glasauer S, Grasso R, Takei Y, Viaud-Delmon I (1999) Dissociation between distance and direction during locomotor navigation. In: Wayfinding behavior (Golledge R, ed), pp 328-348. Baltimore: Johns Hopkins UP.

Blair HT, Sharp PE (1995) Anticipatory head direction signals in anterior thalamus: evidence for a thalamocortical circuit that integrates angular head motion to compute head direction. J Neurosci 15:6260-6270.

Buzsáki G (1986) Generation of hippocampal EEG patterns. In: The hippocampus (Isaacson RL, Pribham KH, eds), pp 137-167. New York: Plenum.

Calton JL, Stackman RW, Goodridge JP, Archey WB, Dudchenko PA, Taube JS (2003) Hippocampal place cell instability after lesions of the head direction cell network. J Neurosci 23:9719-9731.

Csicsvari J, Hirase H, Czurko A, Mamiya A, Buzsaki G (1999) Fast network oscillations in the hippocampal CA1 region of the behaving rat. J Neurosci 19:RC20(1-4).

Czurko A, Hirase H, Csicsvari J, Buzsaki G (1999) Sustained activation of hippocampal pyramidal cells by "space clamping" in a running wheel. Eur J Neurosci 11:344-352.

Ekstrom AD, Meltzer J, McNaughton BL, Barnes CA (2001) NMDA receptor antagonism blocks experience-dependent expansion of hippocampal "place fields." Neuron 31:631-638.

Foster TC, Castro CA, McNaughton BL (1989) Spatial selectivity of rat hippocampal neurons: dependence on preparedness for movement. Science 244:1580-1582.

Gothard KM, Skaggs WE, McNaughton BL (1996) Dynamics of mismatch correction in the hippocampal ensemble code for space: interaction between path integration and environmental cues. J Neurosci 16:8027-8040.

Gothard KM, Hoffman KL, Battaglia FP, McNaughton BL (2001) Dentate gyrus and CA1 ensemble activity during spatial reference frame shifts in the presence and absence of visual input. J Neurosci 21:7284-7292. 
Horii A, Russell NA, Smith PF, Darlington CL, Bilkey DK (2004) Vestibular influences on CA1 neurons in the rat hippocampus: an electrophysiological study in vivo. Exp Brain Res 155:245-250.

Israel I, Berthoz A (1989) Contribution of the otoliths to the calculation of linear displacement. J Neurophysiol 62:247-263.

Israel I, Chapuis N, Glasauer S, Charade O, Berthoz A (1993) Estimation of passive horizontal linear whole-body displacement in humans. J Neurophysiol 70:1270-1273.

Jensen O, Lisman JE (1996) Hippocampal CA3 region predicts memory sequences: accounting for the phase precession of place cells. Learn Mem 3:279-287.

Louie K, Wilson MA (2001) Temporally structured replay of awake hippocampal ensemble activity during rapid eye movement sleep. Neuron 29:145-156.

Markus EJ, Barnes CA, McNaughton BL, Gladden VL, Skaggs WE (1994) Spatial information content and reliability of hippocampal CA1 neurons: effects of visual input. Hippocampus 4:410-421.

Maurer AP, VanRhoads SR, Sutherland GR, Lipa P, McNaughton BL (2005) Self-motion and the origin of differential scaling along the septotemporal axis of the hippocampus. Hippocampus, in press.

McNaughton (1998) The neurophysiology of reminiscence. Neurobiol Learn Mem 70:252-267.

McNaughton BL, Barnes CA, O'Keefe J (1983a) The contributions of position, direction, and velocity to single unit activity in the hippocampus of freely-moving rats. Exp Brain Res 52:41-49.

McNaughton BL, O'Keefe J, Barnes CA (1983b) The stereotrode: a new technique for simultaneous isolation of several single units in the central nervous system from multiple unit records. J Neurosci Methods 8:391-397.

McNaughton BL, Chen LL, Markus EJ (1991) “Dead reckoning,” landmark learning, and the sense of direction: a neurophysiology and computational hypothesis. J Cogn Neurosci 3:191-202.

McNaughton BL, Barnes CA, Gerrard JL, Gothard K, Jung MW, Knierim JJ, Kudrimoti H, Qin Y, Skaggs WE, Suster M, Weaver KL (1996) Deciphering the hippocampal polyglot: the hippocampus as a path integration system. J Exp Biol 199:173-185.

McNaughton BL, Shen J, Skaggs WE, Barnes CA (1997) A new definition of a unitary place field based on phase precession. Soc Neurosci Abstr 23:196.6.

Mehta MR, Barnes CA, McNaughton BL (1997) Experience-dependent, asymmetric expansion of hippocampal place fields. Proc Natl Acad Sci USA 94:8918-8921.

Mittelstaedt H, Mittelstaedt M (1980) Homing by path integration in a mammal. Naturwissenschaften 67:566-567.

O'Keefe J, Nadel L (1979) The hippocampus as a cognitive map. Oxford: Clarendon.

O'Keefe J, Recce ML (1993) Phase relationship between hippocampal place units and the EEG theta rhythm. Hippocampus 3:317-330.

Quirk GJ, Muller RU, Kubie JL (1990) The firing of hippocampal place cells in the dark depends on the rat's recent experience. J Neurosci 10:2008-2017.

Ranck Jr JB (1973) Studies on single neurons in dorsal hippocampal forma- tion and septum in unrestrained rats. I. Behavioral correlates and firing repertoires. Exp Neurol 41:461-531.

Recce ML, O'Keefe J (1989) The tetrode: an improved technique for multiunit extracellular recording. Soc Neurosci Abstr 15:490.3.

Redish AD, Touretzky DS (1997) Cognitive maps beyond the hippocampus. Hippocampus 7:15-35.

Samsonovich A, McNaughton BL (1997) Path integration and cognitive mapping in a continuous attractor neural network model. J Neurosci 17:5900-5920.

Skaggs WE, McNaughton BL, Gothard KM, Markus EJ (1993) An information-theoretic approach to deciphering the hippocampal code. In: Advances in neural information processing systems 5 (Hanson SJ, Cowan JD, Giles CL, eds), pp 1030-1037. San Mateo, CA: Kaufmann.

Skaggs WE, Knierim JJ, Kudrimoti HS, McNaughton BL (1995) A model of the neural basis of the rat's sense of direction. In: Advances in neural information processing systems 7 (Tesauro G, Touretzky D, and Leen T, eds), pp 173-180. Cambridge, MA: MIT.

Skaggs WE, McNaughton BL, Wilson MA, Barnes CA (1996) Theta phase precession in hippocampal neuronal populations and the compression of temporal sequences. Hippocampus 6:149-172.

Stackman RW, Taube JS (1997) Firing properties of head direction cells in the rat anterior thalamic nucleus: dependence on vestibular input. J Neurosci 17:4349-4358.

Stackman RW, Clark AS, Taube JS (2002) Hippocampal spatial representations require vestibular input. Hippocampus 12:291-303.

Taube JS (1995) Head direction cells recorded in the anterior thalamic nuclei of freely moving rats. J Neurosci 15:70-86.

Taube JS, Muller RU, Ranck Jr JB (1990a) Head-direction cells recorded from the postsubiculum in freely moving rats. I. Description and quantitative analysis. J Neurosci 10:420-435.

Taube JS, Muller RU, Ranck Jr JB (1990b) Head-direction cells recorded from the postsubiculum in freely moving rats. II. Effects of environmental manipulations. J Neurosci 10:436-447.

Terrazas A, Krause M, Bohne KM, Poneta KM, Dees JA, McNaughton BL, Barnes CA (2001) The contributions of vestibular and optic flow information to location specific firing of rat hippocampal pyramidal cells. Soc Neurosci Abstr 27:643.14.

Touretzky DS, Redish AD (1996) Theory of rodent navigation based on interacting representations of space. Hippocampus 6:247-270.

Tsodyks MV, Skaggs WE, Sejnowski TJ, McNaughton BL (1996) Population dynamics and theta rhythm phase precession of hippocampal place cell firing: a spiking neuron model. Hippocampus 6:271-280.

Wallenstein GV, Hasselmo ME (1997) GABAergic modulation of hippocampal population activity: sequence learning, place field development, and the phase precession effect. J Neurophysiol 78:393-408.

Wilson MA, McNaughton BL (1993) Dynamics of the hippocampal ensemble code for space. Science 261:1055-1058.

Yamaguchi Y, Aota Y, McNaughton BL, Lipa P (2002) Bimodality of theta phase precession in hippocampal place cells in freely running rats. J Neurophysiol 87:2629-2642.

Zhang K (1996) Representation of spatial orientation by the intrinsic dynamics of the head-direction cell ensemble: a theory. J Neurosci $16: 2112-2126$ 